Pacheco-Salazar, B. y López-Yáñez, J. (2019). "Ella lo provocó": el enfoque de género en la comprensión de lá violencia escolar. Revista de Investigación Educativa, 37(2), 363-678.

DOI: http://dx.doi.org/10.6018/rie.37.2.321371

\title{
“Ella lo provocó”: el enfoque de género en la comprensión de la violencia escolar
}

\section{"She provoked it": a gender-based approach to understanding school violence}

\author{
Berenice Pacheco-Salazar y Julián López-Yáñez \\ *Profesora del Área de Ciencias Sociales y Humanidades. Instituto Tecnológico de Santo Domingo (República Domi- \\ nicana) \\ **Catedrático de Universidad. Departamento de Didáctica y Organización Educativa. Facultad de Educación. Universi- \\ dad de Sevilla (España)
}

\section{Resumen}

El objetivo de este trabajo es analizar la presencia de los estereotipos de género en las dinámicas de violencia escolar. Se realizó un estudio descriptivo con diseño cualitativo en dos centros educativos en República Dominicana, a partir de una muestra de 604 estudiantes entre 9 y 16 años. Las técnicas de levantamiento de información fueron talleres lúdicos-creativos, grupos focales y observación no participante.

Los resultados evidencian que el arraigo a los roles estereotipados de género se constituye en un perpetuador de la violencia escolar. Esto se expresa en la violencia verbal y física y, de manera aún más dramática, en la violencia sexual, de la cual las alumnas siempre son las principales víctimas. Además, la socialización diferenciada produce que manifestaciones de violencia de género en la escuela sean invisibilizadas.

Los hallazgos plantean la necesidad de formar a estudiantes en igualdad de género y educación afectivo-sexual, e integrar el enfoque de género y la coeducación en el desarrollo profesional docente a fin de que los centros educativos puedan desarrollar un abordaje preventivo y no reduccionista de la violencia escolar.

Correspondencia: Berenice Pacheco-Salazar, berenice.pacheco@intec.edu.do, Avenida de Los Próceres \#49, Los Jardines del Norte 10602, Santo Domingo, República Dominicana. Apartado postal 342-9 y 249-2. 
Palabras clave: género, estereotipos de género, violencia escolar, relaciones entre pares, coeducación.

\begin{abstract}
The aim of this research is to analyze the presence of gender stereotypes in the dynamics of school violence. A descriptive study with qualitative design was carried out in two schools in the Dominican Republic, from a sample of 604 students between the ages 9 to 16 years old. The information-gathering techniques included creative play workshops, focus groups, and non-participant observation.

The results demonstrate that the deeply-rooted gender stereotypes constitute a perpetuating support of school violence. This is expressed in verbal and physical violence and, even more severely, in sexual violence, of which female students are always the main victims. Furthermore, differentiated socialization renders manifestations of gender violence in school invisible.

These findings raise the need to train students in gender equality and affective-sexual education, and to integrate a gender-based approach in the professional development of teachers in order to achieve a preventive approach to school violence.

Keywords: gender; gender stereotypes; school violence; peer relationships; gendered education.

\section{Introducción}

La violencia puede ser definida como un comportamiento socialmente aprendido que entraña una intención y necesidad de imposición, control y daño, y que está sustentada en la desigualdad relacional de poder. De manera específica, la violencia escolar es aquella que ocurre o se gesta en el centro educativo, y que lesiona la integridad de docentes y estudiantes, impidiendo, además, el logro de los propósitos educativos.

La violencia escolar debe ser siempre entendida como un reflejo de la que acontece en su contexto social más amplio. Diversos autores han enfatizado la importancia de analizar los factores sociales, familiares y personales que influyen en la ocurrencia de este fenómeno (Casas \& Ortega, 2014; Collel \& Escudé, 2014; Gómez-Ortiz, Del Rey, Viguer \& Solé, 2012). El presente trabajo se enfoca en el abordaje de uno de los más importantes factores sociales en la comprensión de las dinámicas de violencia escolar: el arraigo de los estereotipos de género.
\end{abstract}

\title{
Estereotipos de género y violencia escolar
}

Desde los estudios de género se ha señalado y documentado ampliamente, la necesidad de reflexionar sobre las identidades masculinas y femeninas como constructos sociales y, por tanto, como factores perpetuadores de la violencia social y escolar.

La categoría género remite al conjunto de expectativas y comportamientos sociales que se atribuyen en función del sexo (Ferrer \& Bosch, 2013). Los mismos se concretan en los estereotipos de género, que se entienden como "aquellas generalizaciones no científicas acerca de lo que es propio de cada sexo” (Espín, Martín \& Rodríguez, 2004, p. 209). De esta manera, los estereotipos se conforman por creencias, prescripciones, 
roles y atribuciones que construyen la subjetividad y el comportamiento de los sujetos sociales; y que se transmiten e interiorizan a través de las instancias de socialización como los patrones propios y esperados en hombres y mujeres.

En palabras de Rebollo-Catalán et al. (2012), a través del proceso de socialización, los estereotipos de género son asumidos como parte del autoconcepto y de la forma de relacionamiento social. De este modo, se configuran formas dicotómicas y antagónicas de percibir, sentir, interpretar y convivir entre mujeres y hombres. Por tanto, los estereotipos de género se constituyen en un eje organizador social que genera desigualdad, en tanto otorga diferente valoración de lo femenino subordinado a lo masculino.

A través del denominado currículo oculto, la escuela reproduce y perpetúa los estereotipos de género. Esto afecta, no solo la gestión y la convivencia escolar (GarcíaPérez et al., 2010), sino también los logros académicos del estudiantado (Igbo, Onu \& Obiyo, 2015), y su identidad, autoconcepto y proyecto de vida.

La conformación estereotipada de las identidades masculinas y femeninas es uno de los principales fundamentos de la violencia en todos los espacios sociales, incluido el escolar (McNamara, 2013; Meyer, 2014; Moya \& De Lemus, 2007; Secretaría de Educación Pública \& Unicef, 2009). Estudios previos han documentado la vinculación que existe entre la socialización diferencial, la cultura estereotipada de género y la violencia escolar (Blaya, Debarbieux \& Molina, 2007; Ferrer \& Bosch, 2013; Lomas, 2007).

\section{Objetivo}

Aunque el estudio que da origen a este trabajo aborda la temática de la violencia escolar de manera más amplia, el presente artículo se centra en el objetivo de analizar la presencia de los estereotipos de género en las dinámicas de violencia escolar en República Dominicana.

\section{Método}

El enfoque metodológico de la investigación fue cualitativo, de corte descriptivo y no experimental. La estrategia de investigación fue un estudio de casos múltiples a través del estudio a profundidad de dos centros educativos.

\section{Contexto y participantes}

La investigación fue realizada en dos centros educativos públicos de República Dominicana. Uno de ellos funciona en tanda vespertina (14:00 - 17:00) y cuenta con una matrícula de 274 estudiantes. El segundo centro opera en modalidad de jornada escolar extendida (8:00 - 16:00) y tiene una matrícula de 604 estudiantes. Ambos pertenecen a contextos urbanos marginales y tienen similares condiciones de vulnerabilidad económica.

La identificación de las escuelas se realizó a través del Instituto Dominicano de Evaluación e Investigación de la Calidad Educativa (IDEICE) del Ministerio de Educación de la República Dominicana (MINERD). Junto a los investigadores, se 
localizaron centros de distintas provincias que habían participado previamente en un estudio nacional cuantitativo sobre violencia escolar (IDEICE \& UNIBE, 2014), que contaran con los grados de quinto a octavo y que manifestaran interés por ser parte de la investigación.

En seis centros educativos se realizaron jornadas de presentación del estudio, sin embargo, estos no aceptaron involucrarse alegando la no disponibilidad de tiempo. Posteriormente, se repitió el mismo proceso con tres nuevas escuelas, de las cuales dos accedieron y se constituyeron en los casos del presente trabajo.

Se obtuvo la autorización de estos dos centros educativos para acceder al campo y se acordó con ellos el cronograma de trabajo. De manera informada y voluntaria, participaron 604 estudiantes de quinto a octavo grado, en edades entre los 9 y 16 años, esto es, con un importante nivel de sobreedad escolar según las edades esperadas para dichos grados (véase la tabla 1). En el centro educativo A se trabajó de manera directa con un total de 208 estudiantes, mientras que en el centro B se trabajó con 396 estudiantes, tal y como puede observase en la tabla 2.

Tabla 1

Correspondencia entre grados, nivel educativo y edades esperadas en el sistema educativo dominicano

\begin{tabular}{ccc}
\hline Grados & Nivel educativo & $\begin{array}{c}\text { Edades esperadas } \\
\text { del estudiantado }\end{array}$ \\
\hline $5^{\mathrm{o}}$ & Segundo ciclo del Nivel Primario & 10 \\
$6^{\circ}$ & Segundo ciclo del Nivel Primario & 11 \\
$7^{\mathrm{o}}$ & Primer ciclo del Nivel Secundario & 12 \\
$8^{\mathrm{o}}$ & Primer ciclo del Nivel Secundario & 13 \\
\hline
\end{tabular}

Tabla 2

Estudiantes participantes

\begin{tabular}{rc}
\hline & Estudiantes \\
\hline Centro A & 208 \\
Centro B & 396 \\
Total & 604 \\
\hline
\end{tabular}




\section{Instrumentos}

Para la recolección de información se utilizaron tres técnicas: la observación no participante, los talleres lúdicos-creativos y los grupos focales. El trabajo de campo se desarrolló a través de una inmersión de cuatro meses en cada uno de los centros educativos. Esto, junto a la triangulación de los datos obtenidos de las tres técnicas, aporta confiabilidad, exactitud y consistencia interna a los resultados.

Para la observación no se prefijaron dimensiones de análisis. El registro de la información en el diario se campo se realizó a través de un sistema descriptivo-narrativo abierto, que permitió conocer las opiniones, experiencias y sentidos de interpretación que el estudiantado tiene sobre la problemática investigada. Este proceso de observación no participante fue desarrollado por tres investigadores a fin de minimizar sesgos personales.

Destaca como aspecto innovador del presente estudio, el diseño e implementación de los talleres lúdicos-creativos. Siguiendo los aportes de Paulín et al. (2011), en ellos se trabajó con dinámicas de dibujo, escritura creativa y expresión teatral como dispositivos de recolección de información. Estos talleres además contribuyeron a generar empatía y confianza con el estudiantado, que pudo expresarse sin el condicionamiento de categorías consideradas a priori.

Cada taller contó con la participación de 20 a 25 estudiantes de un mismo grado seleccionados al azar, y de cada uno resultó un documento con las transcripciones y observaciones del taller, así como producciones escritas y visuales del estudiantado.

El análisis de las primeras semanas de observación y de los datos arrojados por los talleres lúdicos-creativos, permitió el establecimiento de un sistema preliminar de categorías que fue utilizado para definir los temas de conversación de los grupos focales. Esto es coherente con los planteamientos de Simons (2011) para quien "vincular estrechamente la entrevista con la observación contribuye a garantizar que nuestras observaciones no sean falsas representaciones" (p. 90).

En los grupos focales se dialogó en torno a diez principales temas, que guiaron el proceso de recogida de datos: caracterización del contexto familiar y comunitario, valoración del clima escolar y del personal docente, valoración sobre la participación estudiantil en la vida y gestión escolar, concepciones sobre qué es violencia escolar, concepciones sobre sus causas y consecuencias, manifestaciones y tipologías de violencia escolar identificadas, principales espacios y momentos de ocurrencia, sujetos implicados y direccionalidades con la que ocurre, estrategias personales e institucionales para el abordaje de la violencia escolar, y propuestas para atender los casos de violencia y contribuir a su prevención.

Se destaca además que, en su mayoría, los grupos focales se desarrollaron con alumnas y alumnos por separado, con la finalidad de propiciar la expresión más libre de sus asunciones y sentimientos. No obstante, también se realizaron algunos grupos focales mixtos. En total, tal y como presenta la tabla 3, se registraron 165 horas de observación, y se realizaron un total de 12 talleres lúdicos-creativos y 45 grupos focales, con participación de 300 y 304 estudiantes respectivamente. 
Tabla 3

Síntesis del trabajo de campo realizado e información recolectada

\begin{tabular}{|l|l|l|}
\hline Técnicas utilizadas & \multicolumn{1}{|c|}{ Información recolectada } & \multicolumn{1}{c|}{ Participantes por centro } \\
\hline $\begin{array}{l}\text { Talleres lúdicos- } \\
\text { creativos }\end{array}$ & $\begin{array}{l}\text { 12 talleres con participación } \\
\text { de } 300 \text { estudiantes }\end{array}$ & $\begin{array}{l}\text { 100 estudiantes del centro A } \\
\text { 200 estudiantes del centro B }\end{array}$ \\
\hline Grupos focales & $\begin{array}{l}\text { 45 grupos focales con partici- } \\
\text { pación de 304 estudiantes }\end{array}$ & $\begin{array}{l}108 \text { estudiantes del centro A } \\
196 \text { estudiantes del centro B }\end{array}$ \\
\hline $\begin{array}{l}\text { Observaciones no } \\
\text { participantes }\end{array}$ & $\begin{array}{l}\text { 165 horas de observación reg- } \\
\text { istradas en diario de campo. }\end{array}$ & $\begin{array}{l}52 \text { horas registradas en el centro A } \\
113 \text { horas registradas en el centro B }\end{array}$ \\
\hline
\end{tabular}

\section{Procedimiento de recogida y análisis de datos}

A través de la inmersión en los centros educativos, la información fue recogida de manera progresiva hasta lograr la saturación teórica.

En coherencia al enfoque cualitativo, el sistema de categorías fue construido de manera inductiva y emergente a través de la escucha activa y análisis de la información, y se utilizó el método comparativo constante para su validación. Además, se llevó a cabo un proceso de validación del sistema de categorías que incluyó la codificación de cuatro grupos focales por parte de los investigadores y tres especialistas externos, a fin de lograr su fiabilidad. Dichos especialistas cuentan con doctorado en educación o psicología social, estudios en el ámbito de género, y experiencia previa de trabajo en estudios cualitativos. Se utilizó el programa informático MAXqda 12 para el análisis de la información.

La intensiva y prolongada inmersión en el campo, la triangulación de tres técnicas de levantamiento de información y la colaboración de tres investigadores externos en la codificación inicial de los datos y en el análisis e interpretación de estos, aportan validez y confiabilidad a los resultados y conclusiones de la investigación.

El presente artículo se centra en aquellas dimensiones relativas a la presencia de los estereotipos de género en las dinámicas de violencia escolar. Las categorías de análisis que son consideradas en el presente trabajo son: causas de la violencia escolar (causas comunitarias, causas familiares, construcción social de género) y manifestaciones y tipologías de la violencia escolar (verbal, física con uso de armas, sexual, expresada en el noviazgo y relaciones de pareja).

El material de campo utilizado para ilustrar los resultados procede de los grupos focales y observaciones, aunque su significado se construyó también utilizando el material obtenido de los talleres.

\section{Resultados}

\section{Estereotipos de género en la comprensión de las causas de la violencia escolar}

En los centros educativos, el estudiantado pone de manifiesto su arraigo a los estereotipos tradicionales de género al momento de expresar sus consideraciones sobre 
cuáles son las causas de la violencia escolar. Por un lado, sus opiniones evidencian una normalización de la conducta violenta como parte de la identidad masculina.

Los varones son así, groseros, violentos. (Alumna $5^{\circ}$ )

Los varones siempre son violentos, siempre. (Alumna $6^{\circ}$ )

A mí me gustan. Me gustan las mujeres bien arregladas, que se ven bien, que sean hermosas, que se vistan bien. (...) Y es como él dijo, las mujeres son para tener hijos y violarlas. (Alumno $5^{\circ}$ )

Es que somos así, eso es ser hombre. Que vean que uno es macho, agresivo. (Alumno $8^{\mathrm{o}}$ )

Por otro lado, estudiantes también expresan la consideración de que las alumnas son provocadoras de la violencia sexual de la cual son víctimas y, por tanto, son merecedoras de las consecuencias de "incitarla".

Es que ellas siempre nos andan provocando, siempre. Enseñan su cuerpo, enseñan la nalga. Nos provocan para que las matemos [sostengan relaciones sexuales]. (Alumno 5 $5^{\mathrm{o}}$ )

Nos provocan. Tienen mucha nalga. Nos viven provocando. Ellas son las culpables. (Alumno $8^{\circ}$ )

No voy a decir que todas somos iguales, pero aqui hay algunas muchachas que les gusta, que les gusta que les agarren todo, que le agarres las tetas, las nalgas y todo. Esas muchachas se lo buscan porque es que viven provocando. (Alumna 5)

\section{Presencia del sexismo en la violencia verbal}

Las amenazas, chismes, apodos e insultos caracterizan el relacionamiento cotidiano del estudiantado en los centros educativos. Estas manifestaciones de violencia verbal, se constituyen a la vez en un factor detonante de violencia física.

Los chismes son considerados como una actividad asociada a lo femenino, propia de las alumnas, según opina el estudiantado. "Las mujeres son las de los chismes siempre", es una frase de una alumna de $8^{\circ}$, que expresa la opinión generalizada encontrada en los centros educativos.

Por otra parte, los apodos o sobrenombres se instauran como mecanismos de vigilancia y control de la identidad y corporalidad entre pares, acorde a los parámetros establecidos por el racismo y el sexismo.

Con relación a esto último, desde los apodos se sancionan aquellos comportamientos alejados a los mandatos tradicionales de género, sirviendo como insultos y ridiculizaciones. En el caso de los alumnos, los apodos están marcados por una fuerte carga de homofobia y heteronormatividad; mientras que en las alumnas, sirven de sanción ante un tipo de comportamiento sexualmente activo que se considera propio de lo masculino. De esta manera, los apodos - en tanto violencia verbal - favorecen la perpetuación de los roles estereotipados de género y, con ello, operan como reproductores cotidianos de la desigualdad. 
A ese le dicen maricón porque camina así, con un andadito, como si fuera una mujer. (Alumno $7^{\circ}$ )

A mí también me viven relajando y me dicen gay. Hay días que no me importa pero hay días que yo quisiera venir con una ametralladora y matar a todo el mundo. (Alumno $5^{\mathrm{o}}$ )

Le dicen pica pollo, porque tiene el toto ripiao [vulva rajada] (risas). (Alumna $7^{\circ}$ ) Ella es marimacho. Le encanta hacer lo de los varones. (Alumno $6^{\circ}$ )

La interpretación y el posterior enjuiciamiento de los comportamientos y prácticas del estudiantado se realizan desde una doble moral sexual. Mientras que la conducta sexualmente activa de las alumnas -expresada, por ejemplo, en el hecho de tener o haber tenido múltiples parejas-, es sancionada y estigmatizada, en los alumnos se asume como algo deseable y propio de su identidad. Esto último, a su vez, constituye un elemento que permanentemente genera competitividad y situaciones de violencia entre las alumnas.

Es que los varones son malos, son tigueres. Se ponen a enamorar a varias muchachas y empieza a tener varias novias, aquí mismo, en la misma escuela. (Alumna $6^{\circ}$ ) En esta escuela las mujeres discuten y pelean porque tienen el mismo marido o tienen el mismo novio. Eso es un pleito [pelea] todos los días. (Alumno 7º)

Yo tengo varias (...). Si una no quiere estar conmigo, quiere la otra. (Alumno $8^{\circ}$ ) Tengo dos [novias] ahora mismo. (Alumno $8^{\circ}$ )

\section{Presencia del sexismo en el uso y porte de armas}

Estudiantes consideran que la violencia física entre pares es muy frecuente, al igual que sucede con la verbal. Ambos admiten preocupación por el porte y uso de armas blancas, las cuales son de uso extendido entre el alumnado para amenazar, para agredir y, en sus propias palabras, "para defensa propia".

Todo el mundo aquí trae su asunto. Hay que defenderse. Uno trae su cuchillito, su navaja. (Alumno 5)

Entre nosotras las mujeres peleamos con cuchillo o con pincho [horquilla]. Yo siempre traigo mis cosas para que no se atrevan a ponerse conmigo. (Alumna $6^{\circ}$ )

No, la directora no se entera. Cuando uno tiene problemas, uno trae su cuchillo y puñal en las medias o en la mochila. Hay que defenderse. Uno no va a ser pendejo. Hay que darse a respetar. (Alumno $8^{\circ}$ )

Es interesante acotar que tanto alumnos como alumnas se encuentran implicados activamente en la violencia física directa. Es usual presenciar episodios de violencia física cruzada entre estudiantes. No obstante, sí parece existir una disimilitud de género en el porte y motivos de uso de las armas blancas.

Mientras los cuchillos y las navajas son utilizados por escolares de ambos sexos, las horquillas (también llamadas invisibles o pinchos) y las agujas son las únicas armas de uso exclusivo por las alumnas, cuya utilidad se vincula fundamentalmente a peleas 
originadas por conflictos por enamorados. Dado que las horquillas se utilizan como parte de los peinados, constituyen elementos de fácil porte en la escuela pues pasan desapercibidos. Estas les sirven para cortar rostros, con la finalidad de así provocar una cicatriz que menoscabe la estética de quien se considera una competidora en las relaciones afectivas.

Aquí hay varias rajadas por pinchos [cortadas por horquillas]. A mí no me gustaría que me toque, por eso no me meto en pleitos [peleas], pero así es que discuten las mujeres. Así y con los moños [halándose el pelo]. (Alumna 5ㅜ)

En la cabeza uno trae los pinchos [horquilla] y si están jodiendo [molestando] a uno, entonces uno se lo saca y raja. Con eso le queda su buena cicatriz. (Alumna 6ㅇ) Lo quitamos lo de arriba [la cabeza de la horquilla] y se afila con la pared o la butaca, y ya con eso no se defiende. Se raja a cualquiera si hay que raja. (Alumna $8^{\circ}$ )

\section{Violencia sexual escolar y ab(uso) del cuerpo femenino}

Alumnas y alumnos evidencian tener interiorizada la violencia como un medio de enamoramiento, y como un elemento intrínseco en las relaciones de noviazgo.

Uno sabe que el varón está enamorado si empieza a joderte [molestarte], si no te deja tranquila, te tira papeles, te empuja... (Alumna $5^{\circ}$ )

Si te quieren enamorar, te empiezan a mandar mandados, a tirarte papeles, te empujan en recreo. (Alumno $8^{\circ}$ )

Si me dio es porque está enamorado de mí. (Alumna $7^{0}$ )

En coherencia con la cultura patriarcal prevaleciente, el trabajo de campo evidenció que el estudiantado tiene interiorizada la noción del cuerpo femenino como objeto sexual cuyo propósito es ofrecer placer a otros. Al respecto, se pudieron identificar cuatro principales manifestaciones de violencia sexual contra las alumnas: ver su ropa interior, tocar y agarrar sus nalgas, senos o vulvas, masturbarse y exhibirse frente a ellas y "darles piquete".

Por parte de los alumnos, resulta cotidiano el interés en querer verles la ropa interior a las alumnas, utilizando para ello, en algunos casos, pequeños espejos o los teléfonos móviles.

Nos levantan la falda así para vernos los pantis [bragas]. (Alumna $7^{\circ}$ )

Los de octavo usan espejos. Los ponen así, abajo, y con eso nos ven debajo de la falda. (Alumna $7^{\mathrm{o}}$ )

Según reportaron las alumnas, sus pares varones les suelen agarrar -en sus propias palabras- "las nalgas, las tetas...". Esta es una práctica recurrente que evidencia la significación del cuerpo femenino como objeto de placer. Durante el trabajo de campo, se constató que algunos alumnos tocaban de manera forzosa a sus compañeras o les besaban, en ocasiones acorralándoles entre varios. 
Eso es siempre. Si uno va a pasar por donde están los varones siempre te quieren hacer algo. Te agarran, te suben la falda, a veces entre varios. (Alumna $5^{\circ}$ )

Sí, te pasan lapiceros por ahí [las nalgas], y te agarran las tetas y ahí también [la vulva]. (Alumna $6^{\circ}$ )

Es que ellos [los alumnos] no conocen otra forma de relajar. Todo es agarrándote las partes. (Alumna $8^{\circ}$ )

Otra práctica usual es la masturbación de los alumnos, lo cual sucede en el patio del recreo y en el aula, inclusive con presencia del profesorado. Además, se manifestó que alumnos -especialmente de $5^{\circ}$ y $6^{0}$ grado- bajan sus pantalones para mostrar sus genitales a las alumnas, lo que se constituye en una acción de exhibicionismo y acoso.

Ellos se agarran su parte [el pene] y te llaman y cuando tu miras te lo enseñan y se empiezan a hacer así [simula masturbación] ahí mismo, en el curso. (Alumna 6ㅇ) Es normal. Lo hacen mucho. En el mismo curso, en el baño o en el patio. (...) A veces lo hacen como para molestar a uno. (Alumna $7^{\circ}$ )

Finalmente, otra manifestación de violencia sexual en las escuelas es el "dar piquete", término que refiere a cuando los alumnos se "pegan" a las alumnas de manera no consentida.

Me hierve la sangre [me molesta] cuando se ponen a darle piquete a uno. (Alumna 5ㅇ) Siempre lo hacen, esa es su gracia. Se te pegan por detrás y te lo pegan [el pene]. (Alumna 6을

A los varones eso es lo que le gusta. Se pegan a atrás de uno y empiezan a dar piquete. (Alumna $7^{\circ}$ )

Por su parte, los alumnos consideran cada una de estas prácticas como algo "común y normal" y, peor aún, como un juego o broma entre pares.

Ellas se quillan [molestan] a veces, pero es divertido. Le damos piquete a las de todos los cursos. (Alumno 5을

Le agarran el culo y todas sus partes. Eso es verdad. No lo vamos a negar ahora, pero eso es un relajo y vaina. (Alumno $6^{\circ}$ )

Sí, sí. Cuando la profesora sale o está en la pizarra, uno lo hace [se masturba]. (Alumno 6 ${ }^{\circ}$ )

Si tienen una falta con los celulares se le toma su foto. Y si tienen su pantalón apretado, eso es también provocando. ¿Cómo uno se va a quedar quieto? Eso es jugando, pero es que también nos provocan. (...) Les agarramos las tetas y sus vainas. (Alumno $8^{\mathrm{o}}$ )

Los resultados del estudio indican que el personal docente no enfrenta a fondo la violencia sexual en el ámbito escolar. Algunos de estos centros han emprendido, en los últimos años, la iniciativa de cambiar el uniforme de las alumnas, solicitándoles que usen pantalones holgados en lugar de faldas. Con esto se pretende disminuir el riesgo de que ellas sean víctimas de violencia sexual por parte de sus compañeros. 
Esto se constituye en una acción de control de la corporalidad femenina y de responsabilización a las alumnas por la violencia de la que puedan ser víctimas. Con esto se dejan impunes y sin sanción a los alumnos que ejercen la violencia, al no considerar acciones preventivas ni de formación.

Aquí nadie usa faldas ya. Dicen que hay que venir con pantalones para evitar situaciones y no provocarlos. (...) La directora ahora solo quiere que uno venga con pantalón. (Alumna $7^{\mathrm{o}}$ )

Tenemos que venir con pantalones. Ahora dicen que no es con falda y que solo nos van a dejar entrar si es con pantalones. (...). Y los pantalones tiene que ser anchos, como si uno fuera una vieja. (Alumna $8^{\circ}$ )

Si las mujeres no quieren provocarnos que vengan con otra ropa, que vengan como monjas. (Alumno 6우).

\section{Discusión y conclusiones}

Los hallazgos de esta investigación muestran que el arraigo a los roles estereotipados de género se constituye en un sostén perpetuador tanto de la violencia escolar -en sus diversas manifestaciones-, como de la desigualdad en el relacionamiento entre mujeres y hombres. Asimismo, la interiorización de estos roles provoca que manifestaciones de violencia de género en la escuela sean naturalizadas e invisibilizadas (Rebollo-Catalán, Mayor-Buzon \& Vico-Bosch, 2018).

La violencia verbal caracteriza la cotidianidad escolar, y los apodos - utilizados como insultos - son su más frecuente expresión, al convertirse en un mecanismo de vigilancia de las normativas y mandatos de género; así como de castigo para quienes los transgreden. Esto coincide con anteriores trabajos realizados en República Dominicana (Fundación Santa María \& Organización de Estados Iberoamericanos para la Educación, la Ciencia y la Cultura, 2008), Venezuela (Rodríguez \& Delgado, 2010) y Uruguay (Baridon \& Martín, 2014), que han concluido que las expresiones más frecuentes de violencia verbal escolar son las amenazas, los insultos y los apodos.

En lo relativo a la violencia física, tanto alumnas como alumnos se encuentran implicados en su ejercicio. Al respecto, investigaciones precedentes (Álvarez-González \& Costa, 2012) explican que las alumnas pueden llegar a desarrollar formas de violencia tradicionalmente asociadas a lo masculino, a fin de emular las situaciones de éxito percibidas en sus pares. Sin embargo, el presente estudio evidencia una diferencia en la forma en que se ejerce la violencia, por ejemplo, a través del uso y porte de armas. Mientras el uso de navajas y cuchillos está extendido en todo el estudiantado, las horquillas (pinchos) y agujas son utilizadas exclusivamente por las alumnas en conflictos vinculados a relaciones amorosas.

A pesar de esto, el alumnado comprende el ejercicio de la violencia como un elemento intrínseco de la identidad masculina, hallazgo coherente con estudios anteriores (Rebollo-Catalán, Vega-Caro \& García-Pérez, 2011). De esta forma, la violencia se asume como un comportamiento esperado y, por tanto, justificado en los alumnos, pero más sancionado en las alumnas a quienes, además, se les considera provocadoras y merecedoras de ser víctimas, en tanto transgresoras de sus roles tradicionales. Esta preconcepción resulta en una mayor tolerancia y permisividad ante el ejercicio de la 
violencia por parte de los alumnos; a partir de lo cual se silencian y minimizan muchos de los episodios de violencia sexual de los cuales las alumnas son las víctimas.

Se ha establecido que el arraigo a los estereotipos de género favorece que los alumnos evadan la responsabilidad de su comportamiento violento y que las y los estudiantes culpabilicen a las víctimas (Bascón, Saavedra \& Arias, 2013; Colás \& Villaciervos, 2007; Díaz-Aguado \& Martínez, 2014; Rebollo-Catalán et al., 2018; Ruiz-Repullo, 2016). Además, se ha documentado ampliamente que la variable género es determinante en la manera en que se interpretan los actos violentos (Estévez, Murgui \& Musitu, 2009; García-Pérez et al. 2011; Ortega, Sánchez, Ortega-Rivera, Nocentini, \& Menesini, 2010; Velasco \& Álvarez-González, 2015). Todo esto revela asimilación de procesos de desplazamiento de responsabilidad y desconexión moral (Ortega, 2008; Sánchez, Ortega \& Menesini, 2012).

Por otro lado, el discurso y las prácticas de estudiantes reflejan la interiorización de la violencia como vía para establecer relaciones afectivas, lo que se expresa en los procesos de cortejo y enamoramiento. Otras investigaciones han señalado que, durante la adolescencia, la internalización de los roles estereotipados de género puede derivar en que comportamientos violentos lleguen a considerarse como expresiones de afecto, en tanto se admite la violencia como elemento integrador de la identidad masculina, y el sacrificio y sufrimiento de la femenina (Díaz-Aguado, 2009; Laporte, Jiang, Pepler \& Chamberland, 2011; Ruíz-Repullo, 2016; Sánchez, Galicia \& Robles, 2013).

Resulta preocupante la recurrencia con la que suceden episodios de violencia y acoso sexual en las escuelas, situación que coincide con otros estudios realizados en Europa y América Latina (Benbenishty et al., 2015; Díaz-Aguado, 2009; Piñero, Arense, López \& Torres, 2014).

En este contexto, el cuerpo femenino queda configurado como un objeto dócil, disponible para el placer de otros. Esto se expresa claramente en las prácticas cotidianas de los alumnos que pretenden ver, tocar y disfrutar del cuerpo de sus compañeras sin su consentimiento, y asumiéndolo como una "broma". De esta forma, la posesión del cuerpo femenino se constituye en un procedimiento de domesticación y dominación que, desde la satisfacción del deseo sexual, construye y valida la masculinidad hegemónica frente a los pares. Asimismo, las acciones de masturbación y exhibicionismo en el aula se convierten en manifestaciones de violencia y acoso sexual. Las mismas tienen su raíz en la construcción estereotipada de la masculinidad y una expresión sexual distorsionada que le coloca en un rol activo-dominante, tal y como plantean Rebollo-Catalán et al. (2018).

La violencia escolar está sustentada e intrincada con la violencia de género y la masculinidad hegemónica, expresada en el afán masculino de ejercer dominio y autoridad sobre las mujeres, en este caso de las alumnas (Delgado et al. 2007; Díaz-Aguado, 2009). De este modo, la violencia sexual emerge como la única manifestación de violencia escolar donde las principales víctimas siempre son las alumnas (Falla, Alós, Moriana \& Ortega, 2012; Yang \& Salmivalli, 2013), en tanto su direccionalidad está marcada por la construcción estereotipada de las identidades femeninas y masculinas.

En este contexto, los centros escolares se convierten en una instancia social de perpetuación y legitimación de las desigualdades sociales y de género (Bolaños \& Jiménez-Cortés, 2007; Martín, 2016; Rebollo-Catalán et al., 2011; Viguer \& Solé, 2012), 
donde el propio alumnado opera como agente de vigilancia y control que validan los roles estereotipados de género.

Se hace necesario sensibilizar y formar en igualdad de género y educación afectivosexual al estudiantado, de manera que puedan repensar su propio sistema de creencias y valores, y deconstruir la socialización desigual y estereotipada. Asimismo, el enfoque de género y la coeducación han de ser incorporadas como eje transversal del desarrollo profesional docente, a fin de que la escuela pueda desarrollar un abordaje preventivo y no reduccionista de la violencia escolar, y aportar al desarrollo integral de alumnos y alumnas, al margen de los estereotipos de género y desde sus propias potencialidades. Todo esto se constituye en un elemento clave para garantizar el derecho de cada niño, niña, adolescente y joven a disfrutar de una educación de calidad y libre de violencia.

\section{Referencias}

Álvarez-González, B., \& Costa, A. D. (2012). Adiós a las diferencias de género. Una nueva perspectiva en la investigación de sexo y género. Educación XX1, 15(1), 61-86. doi: https://doi.org/10.5944/educxx1.15.1.150

Baridon, D., \& Martín, G. (2014). Violencia escolar en estudiantes de educación media. Ciencias Psicológicas, 8(2), 173-183. Recuperado de http://www.scielo.edu.uy/scielo. php?script=sci_abstract\&pid=S1688-42212014000200007\&lng=pt\&nrm=iso

Bascón, M., Saavedra, J., \& Arias, S. (2013). Conflictos y violencia de género en la adolescencia. Análisis de estrategias discursivas y recursos para la coeducación. Profesorado. Revista de Currículum y Formación de Profesorado, 17(1), 289-307.

Benbenishty, R., López, V., Astor, A., Gilkman, H., Raz, T., Ratner, D., Bilbao, M., \& Rafaeli, T. (Mayo, 2015). Comparing school violence in Israel and Chile. En Ministerio de Educación del Perú y Observatoire International de la Violence á l’Ecole (Presidencia), Congreso Mundial Sobre Violencia Escolar. Congreso llevado a cabo en Lima, Perú.

Blaya, C., Debarbieux, E. \& Molina, B. (2007). La violencia hacia las mujeres y hacia otras personas percibidas como distintas a la norma dominante: el caso de los centros educativos. Revista de Educación, 342, 61-81

Bolaños, L. M., \& Jiménez-Cortés, R. (2007). La formación del profesorado en género. Revista de Investigación Educativa, 25(1), 77-95.

Colás, P., \& Villaciervos, P. (2007). La interiorización de los estereotipos de género en jóvenes y adolescentes. Revista de Investigación Educativa, 25(1), 35-58.

Collel, J., \& Escudé, C. (2014). Una aproximación al fenómeno del acoso escolar. En R. Bisquerra (Coord.), Prevención del acoso escolar con educación emocional (pp. 15-42). Bilbao: Desclée de Breouwer.

Delgado, C., Iraegui, A., Marquina, L., Martín, M., Palacios, B., Plaza, J., Sendín, P., Pérez, M., Revuelta, F., \& Sánchez, M. (2007). Patrones de masculinidad y feminidad asociados al ciclo de la violencia de género. Revista de Investigación Educativa, 25(1), 187-217. 
Díaz-Aguado, M., \& Martínez, R. (2014). Types of adolescent male dating violence against women, self-esteem, and justification of dominance and aggression. Journal of Interpersonal Violence, 30(15), 2636-2658. doi: https://doi.org/10.1177/0886260514553631

Díaz-Aguado, M. (2009). Convivencia escolar y prevención de la violencia de género desde una perspectiva integral. Revista CEE Participación Educativa, 11, 59-72.

Espín, J., Martín, M.A., \& Rodríguez, M. (2004). Análisis del sexismo en la publicidad. Revista de Investigación Educativa, 22(1), 203-231.

Estévez, E., Murgui, S., \& Musitu, G. (2009). Psychosocial adjustment in bullies and victims of school violence. European Journal of Psychology of Education, 24(4), 473- 483. doi: https://doi.org/10.1007/BF03178762

Falla, D., Alós, F., Moriana, J. y Ortega, R. (2012). La violencia entre estudiantes según el profesorado en los Centros de Educación Especial de Córdoba. Aula Abierta, 40(1), 3-14.

Ferrer, V., \& Bosch, E. (2013). Del amor romántico a la violencia de género. Para una coeducación emocional en la agenda educativa. Profesorado. Revista de Curriculum y Formación de Profesorado, 17(1), 105-122.

Fundación Santa María, \& Organización de Estados Iberoamericanos para la Educación, la Ciencia y la Cultura (OEI) (2008). Estudio de Convivencia Escolar en la República Dominicana. Santo Domingo: Fundación SM, OEI.

García-Pérez, R., Rebollo-Catalán, A., Buzón-García, O., González-Piñal, R., BarragánSánchez, R., \& Ruíz-Pinto, E. (2010). Actitudes del alumnado hacia la igualdad de género. Revista de Investigación Educativa, 28(1), 217-232.

García-Pérez, R., Rebollo-Catalán, A., Vega-Caro, L., Barragán-Sánchez, R., BuzónGarcía, O., \& Piedra, J. (2011). El patriarcado no es transparente: competencias del profesorado para reconocer desigualdad. Cultura y Educación, 23(3), 385-397. doi: https://doi.org/10.1174/113564011797330298

Gómez-Ortiz, O., Del Rey, R., Casas, J., \& Ortega, R. (2014). Parenting styles and bullying involvement. Cultura y Educación, 26(1), 132-158. doi: https://doi.org/10.1080 /11356405.2014.908665

Igbo, J., Onu, V., \& Obiyo, N. (2015). Impact of gender stereotype on secondary school students` self-concept and academic achievement. Sage Open, 5(1), 1-10. doi: https:// doi.org/10.1177/2158244015573934

Instituto Dominicano de Evaluación e Investigación Educativa (Ideice) \& Universidad Iberoamericana (Unibe). (2014). Estudio de prevalencia, tipología y causas de la violencia en los centros educativos de básica y media de la República Dominicana. Santo Domingo: Ideice y Unibe.

Laporte, L., Jiang, D., Pepler, D., \& Chamberland, C. (2011). The relationship between adolescents' experience of family violence and dating violence. Youth $\mathcal{E}$ Society, 43(1), 3-27. doi: https://doi.org/10.1177/0044118X09336631

Lomas, C. (2007). ¿La escuela es un infierno? Violencia escolar y construcción cultural de la masculinidad. Revista de Educación, 342, 83-101

Martín, E. (2016) Gender differences in social reputation and peer rejection at school. Cultura y Educación, 28(3), 539-564. doi: https://doi.org/10.1080/11356405.2016.1196899

McNamara, B. (2013). Bullying and students with disabilities. Strategies and techniques to create safe learning environment for all. California: Corwin. 
Meyer, E. (2014). New solutions for bullying and harassment: a post-structural, feminist approach. En R. May, \& D.M. Sondergaard (Eds.), School Bullying. New theories in context (pp. 209-239). New York: Cambridge University Press.

Moya, M., \& De Lemus, S. (2007). Puño de hierro en guante de terciopelo. Diferencias de poder, sexismo y violencia de género. En S. Yubero, E. Larrañaga, \& A. Blanco (Coords.). Convivir con la violencia (pp. 99-116). Cuenca, España: Servicio de Publicaciones de la Universidad de Castilla-La Mancha.

Ortega, R. (2008). Malos tratos entre escolares: de la investigación a la intervención. Madrid: Ministerio de Educación, Política Social y Deporte.

Ortega, R., Sánchez, V., Ortega-Rivera, J., Nocentini, A., \& Menesini, E. (2010). Peer sexual harassment in adolescent girls: A cross-national study (Spain-Italy). International Journal of Clinical and Health Psychology, 10(2), 245-264.

Paulín, H., Tomasini, M., D’Aloisio, F., López, C., Rodigou, M. \& García, G. (2011). La representación teatral como dispositivo de investigación cualitativa para la indagación de sentidos sobre la experiencia escolar con jóvenes. Psicoperspectivas. Individuo y sociedad, 10(2), 134-155.

Piñero, E., Arense, J., López, J., \& Torres, A. (2014). Incidencia de la violencia y victimización escolar en estudiantes de educación secundaria obligatoria en la Región de Murcia. Revista de Investigación Educativa, 32(1), 223-241. doi: https://doi.org/10.6018/ rie.32.1.154251

Rebollo-Catalán, A., Vega-Caro, L., \& García-Pérez, R. (2011). El profesorado en la aplicación de planes de igualdad: conflictos y discursos en el cambio educativo. Revista de Investigación Educativa, 29(2), 311-323.

Rebollo-Catalán, A., Piedra, J., Sala, A., Sabuco, A., Saavedra, J, \& Bascón, M. (2012). La equidad de género en la educación: análisis y descripción de buenas prácticas educativas. Revista de Educación, 358, 129-152. doi: https://doi.org/10.4438/1988592X-RE-2012-358-186

Rebollo-Catalán, A., Mayor-Buzon, V., \& Vico-Bosch, A. (2018). La otra cara de la luna: la violencia invisible contra las mujeres en las redes sociales. En T. Donoso-Vázquez, \& A. Rebollo-Catalán (Coords.), Violencias de género en entornos virtuales (pp. 105120). Barcelona: Octaedro.

Rodríguez, A., \& Delgado, G. (2010). Estudio de expresiones de violencia escolar entre estudiantes de escuelas básicas venezolanas. Revista de Investigación Educativa, 34(70), 71-83.

Ruiz-Repullo, C. (2016). Voces tras los datos. Una mirada cualitativa a la violencia de género en adolescentes. Sevilla: Instituto Andaluz de la Mujer.

Sánchez, V., Ortega, R., \& Menesini, E. (2012). La competencia emocional de agresores y víctimas de bullying. Anales de psicología, 28(1), 71-82.

Sánchez, A., Galicia, I., \& Robles, F. (2013). Relaciones entre estilos de amor y violencia en adolescentes. Psicología desde el Caribe, 30(2), 211-235.

Secretaría de Educación Pública (SEP), \& Unicef. (2009). Informe nacional sobre violencia de género en la Educación Básica en México. Ciudad México: Secretaría de Educación Pública de los Estados Unidos Mexicanos y Unicef.

Simons, H. (2011). El estudio de caso: teoría y práctica. Madrid: Morata. 
Velasco, M. \& Álvarez-González, B. (2015). Perfiles y percepciones de género en violencia escolar. Revista de Investigación Educativa, 33(1), 211-231. doi: https://doi. org/10.6018/rie.33.1.195891

Viguer, P. \& Solé, N. (2012). Escuela e iguales como contextos de socialización en valores y convivencia: una investigación participativa a través de un debate familiar. Cultura y Educación, 24(4), 475-487. doi: https://doi.org/10.11144/Javeriana. upsy13-5.1pfa

Yang, A. y Salmivalli, C. (2013). Different forms of bullying and victimization: Bullyvictims versus bullies and victims. European Journal of Developmental Psychology, 10(6), 723-738. doi: https://doi.org/10.1080/17405629.2013.793596

Fecha de recepción: 12 de febrero de 2018.

Fecha de revisión: 19 de febrero de 2018.

Fecha de aceptación: 3 de julio de 2018. 